\title{
Efeito do exercício físico nos fatores de risco de doenças crônicas em mulheres obesas
}

\author{
Silvia Vieira da Silva Rocca', Julio Tirapegui ${ }^{1}$, Camila Maria de Melo', \\ Sandra Maria Lima Ribeiro ${ }^{2}$
}

\author{
${ }^{1}$ Departamento de Alimentos e Nutrição Experimental, Faculdade de Ciências Farmacêuticas, \\ Universidade de São Paulo, ${ }^{2}$ Escola de Artes, Ciências e Humanidades, Universidade de São Paulo
}

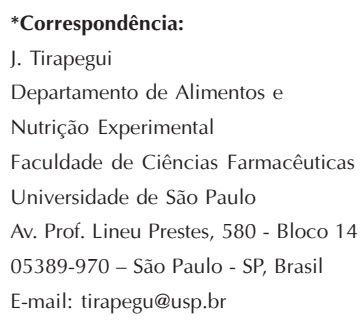

O presente estudo teve por objetivo avaliar os efeitos do exercício fisico em um programa de emagrecimento. Durante 12 semanas, 22 mulheres obesas (IMC>30 kg/m²) foram submetidas a um programa de exercicios fisicos. Ao início e ao final do programa foram avaliados: IMC, circunferências da cintura (CC) e do quadril (CQ), e a relação cinturalquadril (RCQ); composição corporal por DEXA; hemoglobina, eritrócitos, colesterol total, $H D L, L D L$, triacilgliceróis e glicose; potência aeróbia. Ao final do programa, os valores de potência aeróbia, hemácias e eritrócitos foram significativamente elevados, comprovando os efeitos do treinamento. Nos valores de antropometria e composição corporal, apenas os valores relacionados à gordura visceral (CC, $C Q$ e RCQ) sofreram redução significativa. $O$ exercício mostrou-se um importante coadjuvante nos processos de emagrecimento, não por promover redução da massa corporal total, mas em diminuir fatores de risco para o desenvolvimento de doenças crônicas.

\section{INTRODUÇÃO}

No Brasil, assim como em vários outros países em desenvolvimento, a obesidade tem aumentado significativamente nas duas últimas décadas. Dados do Instituto Brasileiro de Geografia e Estatística (IBGE) revelam que $41,1 \%$ dos homens e $40,0 \%$ das mulheres apresentam sobrepeso e $8,9 \%$ e $13,1 \%$, respectivamente, apresentam obesidade (IBGE, 2002-2003; Uauy, Albala, Kain, 2001).

A obesidade é caracterizada pelo acúmulo de gordura corporal, em decorrência da somatória de fatores genéticos e ambientais (ADA, 2002). É considerada preocupante por razões de ordem social, psicológica e metabólica, estando atrelada ao desenvolvimento de comorbidades como diabetes, hipertensão e doenças cardiovasculares. Estudos recentes comprovam que a caracterização do risco de doenças guarda correlação não somente com a quantidade de gordura total no corpo, mas principalmente com o tipo de distribuição corporal dessa gordura (Hedley et al., 2004; Uauy et al., 2001). A obesidade é caracterizada como "superior" ou "central", quando a maior parte da gordura encontra-se na região do tronco, ou ainda "inferior" quando a gordura está localizada principalmente abaixo da cintura, na região glúteo- 
femural. Normalmente esses valores são determinados por medidas como a relação entre as circunferências da cintura e do quadril, ou ainda a circunferência da cintura ou do abdômen (Okura et al., 2002).

É bem estabelecido que modestas perdas de massa corporal total (5 a 10\%) são relevantes para a diminuição do risco de doenças crônicas (Rippe, 1998; ACSM, 2001; ADA, 2002). Barbato et al. (2006) verificaram que uma redução superior a $5 \%$ da massa corporal diminui a circunferência da cintura, glicemia de jejum, colesterol total e LDL-colesterol em indivíduos obesos. Modificações na dieta associada com exercício físico favorecem a perda de massa corporal total, além de reduzir a obesidade abdominal e melhorar a tolerância à glicose (Monteiro, Riether, Burini, 2004).

A maioria dos estudos que avalia efeitos da perda de massa corporal associa restrição alimentar com exercícios físicos. Kempen, Saris e Westerterp (1995) realizaram um estudo de 8 semanas, onde foram constituídos dois grupos: um que realizava somente dieta restritiva e outro que somou a mesma dieta a um programa de exercícios físicos. Os autores verificaram que houve uma diminuição significativa na massa corporal total, na gordura e na massa magra corporal em ambos os grupos. Porém o grupo que realizou atividades físicas associadas à restrição alimentar perdeu em média $2,3 \mathrm{~kg}$ de gordura a mais do que o grupo que fez apenas a dieta.

Sendo assim, o objetivo do presente estudo foi avaliar alterações ocorridas nas variáveis bioquímicas e metabólicas relacionadas ao risco de doenças crônicas em mu- lheres obesas, após um programa de exercícios físicos, mantendo inalterada a ingestão dietética.

\section{MATERIAL E MÉTODOS}

\section{Casuística}

O recrutamento de voluntárias ocorreu a partir da mídia local (jornais regionais e Internet). O fluxo desde o recrutamento até a finalização do projeto encontra-se descrito na Figura 1. Como critérios de inclusão, foram selecionadas mulheres com idade entre 25 e 50 anos, não menopausadas, com índice de massa corpórea (IMC) maior que $30 \mathrm{~kg} / \mathrm{m}^{2}$ e saudáveis. Foram considerados como critérios de exclusão o uso de algum medicamento que interferisse na taxa metabólica, disfunções da glândula tireóide ou ainda a participação em outro programa para perda de peso. Todas as avaliadas assinaram um termo de consentimento livre e esclarecido e o estudo foi aprovado pelo Comitê de Ética em Pesquisa da Faculdade de Ciências Farmacêuticas da Universidade de São Paulo.

\section{Avaliações}

Foram avaliados a massa corporal total e a estatura (balança de mola com precisão de 0,1g Filizola ${ }^{\circledR}$ e estadiômetro Secca ${ }^{\circledR}$ com precisão de $0,1 \mathrm{~mm}$, respectivamente) para posterior cálculo do Índice de Massa Corpórea (IMC). Este valor serviu de referência para classificação das mulheres como obesas (WHO, 1997).

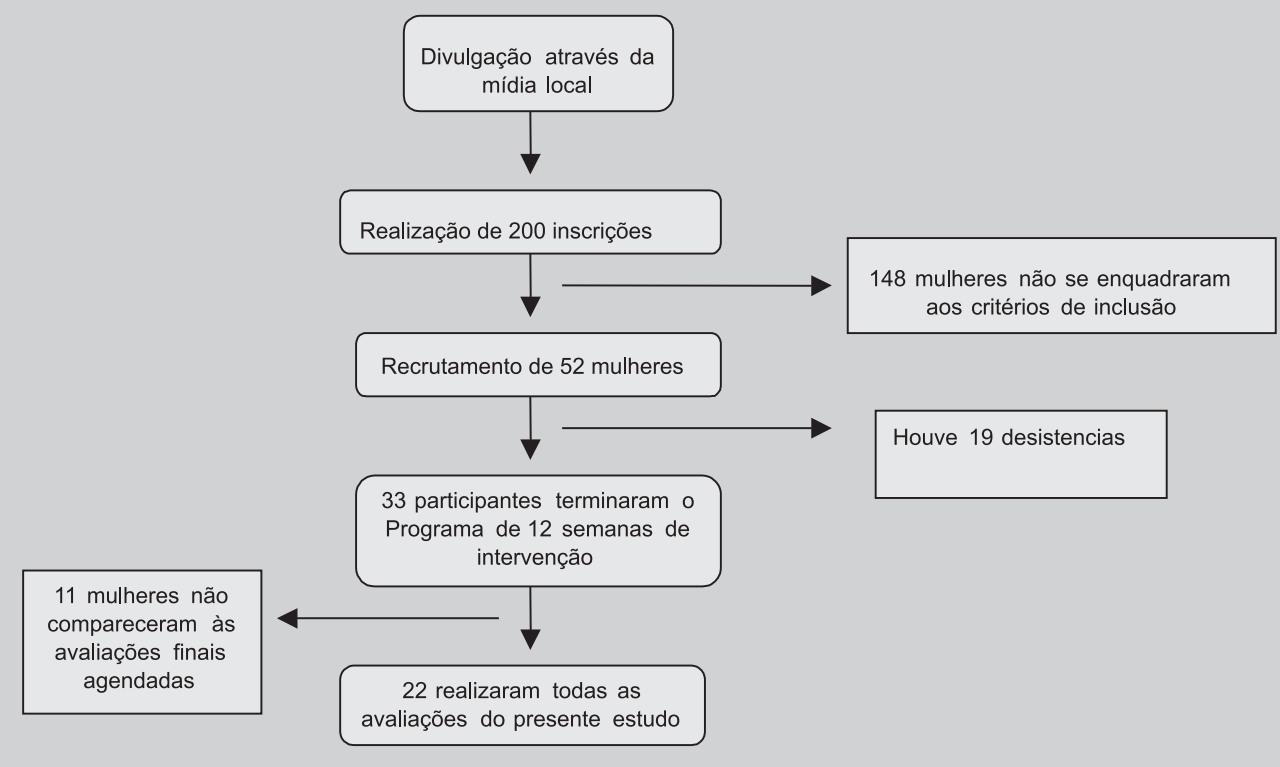

FIGURA 1 - Esquema de recrutamento e desistências da amostra do estudo. 
Acomposição corporal foi determinada por dual-energy $X$-ray absorptiometry (DEXA) realizado por varredura transversal, da cabeça até os pés (Pietrobelli et al., 1996; Lunarß). Para essa análise, as mulheres se dirigiram ao laboratório, foram acomodadas em uma maca em decúbito dorsal, permanecendo imóveis durante aproximadamente 30 minutos, enquanto era realizado o rastreamento de corpo inteiro. Foram consideradas as seguintes variáveis: massa corporal adiposa e massa corporal magra (em valores relativos à massa corporal total), conteúdo e densidade mineral ósseos.

Foram tomadas as circunferências da cintura (CC) e do quadril (CQ), e também calculada a relação cintura/ quadril (RCQ), com uma fita métrica inextensível e inelástica. A circunferência da cintura foi medida no ponto médio entre a costela inferior e a crista ilíaca, e a circunferência do quadril foi mensurada no maior perímetro sobre o trocânter (WHO, 1997).

A pressão arterial foi medida com um esfignomanômetro de mercúrio (Sanny ${ }^{\circledR}$ ) utilizando método auscultatório, após oito a dez minutos de repouso na posição sentada. Foram utilizados manguitos adequados ao diâmetro do braço das avaliadas.

Foram coletadas amostras de sangue nas primeiras horas do dia (entre 7:00 e 9:00h), após período de jejum de 8 a 12 horas, e sem a realização de atividade física intensa no dia anterior. O sangue foi coletado em tubos com anticoagulante para a realização do hemograma completo, através do método automático. Esse método se baseia no uso de três técnicas que avaliam as variações de impedância do fluxo elétrico ou da dispersão de luz produzida pelas diferentes células. Para determinação dos lipídeos e glicemia plasmáticos, o sangue foi centrifugado e apenas o plasma foi utilizado. Foram avaliados: colesterol total, HDL-colesterol, triacilgliceróis e glicose plasmática através de métodos enzimático-colorimétrico (Labtest $\left.{ }^{\circledR}\right)$. O LDL-colesterol foi determinado através da Fórmula de Friedewald: LDLColesterol $=$ colesterol total $-(\mathrm{HDL}+$ triacilgliceróis/5). Os Índices de Castelli I e II (Castelli et al., 1986) foram calculados da seguinte forma: Índice I - divisão da concentração de colesterol total pela concentração de HDL-c (CT/HDLc); Índice II - concentração de LDL-c dividido pela concentração de HDL-c (LDL-c/HDL-c).

A potência aeróbia foi avaliada por meio de teste ergoespirométrico (utilizando o cicloergômetro Biotec 2100, CEFISE $\AA$ e o sistema metabólico computadorizado VO2000, Imbrasport $\left.{ }^{\circledR}\right)$. As avaliadas foram orientadas a comparecer ao laboratório com vestimenta adequada para a realização do teste, realizar uma refeição leve com cerca de duas horas de antecedência e não realizar nenhum exercício físico nas últimas 24 horas. Após questionamentos sobre a história clínica e a realização de exame físico, procedeu-se a monitorização eletrocardiográfica. A seguir, foram orientadas quanto ao método e foi realizada uma breve simulação do teste. Durante o teste foi utilizada uma escala de percepção subjetiva do esforço (Borg, 1982). O protocolo de teste realizado foi do tipo rampa com carga inicial de 30 watts e incremento de 10 watts a cada minuto até a exaustão do indivíduo, momento que foi considerado $\mathrm{VO}_{\text {2pico }}$ (consumo pico de oxigênio). A velocidade foi de $40 \mathrm{rpm}$ durante todo o teste e a duração foi de 8 a 12 minutos.

\section{Programa de atividade física}

As mulheres foram submetidas a um programa de exercícios físicos durante três meses, três vezes por semana com duração de uma hora, sendo trinta minutos de exercício aeróbio e trinta minutos de exercício resistido. Nas primeiras duas semanas foram executadas sessões de adaptação, foi avaliado número máximo de repetições (nRM) para o exercício resistido e o teste ergoespirométrico. O protocolo de exercício aeróbio consistiu de caminhada em esteira ergométrica a $70 \%$ do $\mathrm{VO}_{2 \text { pico }}$, monitorado pela Freqüência Cardíaca (FC) correspondente (freqüêncímetro Polar $\left.{ }^{\circledR}\right)$. O exercício resistido foi executado utilizando uma combinação de pesos livres e aparelhos específicos de musculação. Para cada exercício foram realizadas três séries de 15 repetições a uma intensidade entre $60-70 \%$ do número de repetições máximas (nRM). Foram executados oito exercícios em aparelhos específicos (remada sentada, puxada, supino reto, leg press, extensão de joelhos, flexão de joelhos, flexão plantar e extensão de cotovelo) e quatro exercícios com pesos livres (abdução-adução com flexão de ombro na posição deitada, desenvolvimento frontal, desenvolvimento lateral e flexão de antebraço).

As mulheres foram orientadas a não realizar mudanças bruscas na rotina alimentar durante o programa. Para controle dessa variável, as participantes preencheram três diários alimentares, em dias não consecutivos, no início e no final do programa. Para padronização das medidas e para que se pudesse avaliar a ingestão alimentar com o menor erro possível, foi realizado um treinamento prévio com as mulheres, que foram orientadas a anotar todos os alimentos e bebidas consumidos, com o máximo de detalhamento possível, utilizando medidas caseiras. Os diários foram analisados quantitativamente pelo cálculo de energia (kcal) e proporção de macronutrientes, utilizandose o software Nutri-UNIFESP.

\section{Análise dos dados}

Após a constatação da normalidade dos dados a partir do teste de Kolmogorov-Smirnov, procedeu-se a análi- 
se descritiva dos dados iniciais. As comparações entre os resultados iniciais e finais foram feitas por meio do teste " $\mathrm{t}$ " pareado. Algumas variáveis foram também submetidas ao teste de correlação de Pearson. Para todas as análises foi utilizado o software SPSS para Windows versão 13.0, adotando-se a significância de 5\% ou p $<0,05$.

\section{RESULTADOS}

A idade média das 22 mulheres avaliadas foi de 41,82 $\pm 7,70$ (valor mínimo de 25 e máximo de 55 anos). As Tabelas I e II descrevem as variáveis antropométricas e de composição corporal antes a após o programa.

TABELA I - Medidas antropométricas antes e após o programa $(n=22)$.

\begin{tabular}{lcc}
\hline & $\begin{array}{c}\text { Valores iniciais } \\
(\text { média } \pm \mathrm{DP})\end{array}$ & $\begin{array}{c}\text { Valores finais } \\
\text { (média } \pm \mathrm{DP})\end{array}$ \\
\hline Peso $(\mathrm{kg})$ & $95,30 \pm 15,90$ & $94,61 \pm 16,05$ \\
Estatura $(\mathrm{cm})$ & $162,14 \pm 7,97$ & $162,14 \pm 7,97$ \\
$\mathrm{IMC}\left(\mathrm{kg} / \mathrm{m}^{2}\right)$ & $36,04 \pm 3,97$ & $35,78 \pm 4,05$ \\
$\mathrm{CC}(\mathrm{cm})$ & $103,17 \pm 9,98$ & $99,38 \pm 9,68 *$ \\
CQ $(\mathrm{cm})$ & $123,39 \pm 10,66$ & $121,41 \pm 10,40 *$ \\
RCQ & $0,84 \pm 0,08$ & $0,82 \pm 0,07 *$ \\
\hline
\end{tabular}

* diferença com valor de $\mathrm{p}<0,05$; IMC: índice de massa corporal; CC: circunferência da cintura; CQ: circunferência do quadril; RCQ: relação cintura/quadril.

A Tabela III apresenta as alterações bioquímicas e hematológicas antes e após o programa.

A Tabela IV apresenta as variáveis de pressão arterial e de potência aeróbia antes e após o programa.

Com relação à ingestão alimentar, no início do programa as mulheres consumiam 1909,93 $\pm 443,92 \mathrm{kcal} / \mathrm{dia}$. Ao final do programa, o consumo foi de 1857,23 $\pm 463,12$
TABELA II - Composição Corporal avaliada por DEXA antes e após o programa $(\mathrm{n}=22)$.

\begin{tabular}{lcc}
\hline & $\begin{array}{c}\text { Valores iniciais } \\
(\text { média } \pm \mathrm{DP})\end{array}$ & $\begin{array}{c}\text { Valores finais } \\
(\text { média } \pm \mathrm{DP})\end{array}$ \\
\hline $\mathrm{CMO}(\mathrm{g})$ & $1053,09 \pm 146,15$ & $1040,09 \pm 160,66$ \\
$\mathrm{DMO}\left(\mathrm{g} / \mathrm{cm}^{2}\right)$ & $1,23 \pm 0,08$ & $1,23 \pm 0,08$ \\
MCA $(\%)$ & $44,66 \pm 3,07$ & $45,08 \pm 3,55$ \\
MCM Total $(\%)$ & $53,37 \pm 3,08$ & $52,85 \pm 3,19$
\end{tabular}

* diferença com valor de $\mathrm{p}<0,05$; CMO: conteúdo mineral ósseo; DMO: densidade mineral óssea; MCA: massa corporal adiposa; MCM: massa corporal magra.

kcal, e a diferença entre valores iniciais e finais não foi significativa $(\mathrm{p}=0,672)$.

Verificou-se que no início do programa a medida de circunferência da cintura correlacionou-se inversamente com o consumo máximo de oxigênio $(r=-0,52 ; \mathrm{p}=0,013)$. A relação cintura-quadril (RCQ) correlacionou-se com a fração lipídica HDL-colesterol $(r=-0,45 ; p=0,038)$. Porém, ao final do programa, essas correlações não foram significantes.

\section{DISCUSSÃO}

O presente estudo avaliou alterações em algumas variáveis antropométricas, de composição corporal, bioquímicas e fisiológicas, após um programa de exercícios físicos em mulheres obesas, com três meses de duração.

Pode-se comprovar que as mulheres atenderam apropriadamente ao programa de exercícios, uma vez que, ao final do programa houve um aumento significativo no $\mathrm{VO}_{2 \text { pico }}$. O consumo pico de oxigênio $\left(\mathrm{VO}_{2 \text { pico }}\right)$, que é correlacionado com o consumo máximo de oxigênio, reflete o condicionamento cardiovascular e a capacidade corporal de transportar e utilizar o oxigênio (Paffenbarger et al., 1993; Foss, Keteyian,

TABELA III - Variáveis bioquímicas e hematológicas antes e após o programa (n=22).

\begin{tabular}{lcc}
\hline & Valores iniciais (média \pm DP) & Valores finais (média \pm DP) \\
\hline Colesterol total (mg/dL) & $185,77 \pm 32,68$ & $195,09 \pm 27,48 *$ \\
HDL-colesterol (mg/dL) & $39,47 \pm 9,56$ & $43,40 \pm 10,05 *$ \\
LDL-colesterol (mg/dL) & $116,81 \pm 26,90$ & $119,90 \pm 21,81$ \\
Triacilgliceróis (mg/dL) & $147,51 \pm 59,18$ & $158,98 \pm 54,52$ \\
Glicemia (mg/dL) & $100,15 \pm 18,02$ & $93,73 \pm 14,54 *$ \\
Índice Castelli I (CT/HDL) & $4,86 \pm 1,01$ & $4,68 \pm 1,08$ \\
Índice Castelli II (LDL/HDL) & $3,08 \pm 0,88$ & $2,91 \pm 0,88$ \\
Hemoglobina (g/dL) & $13,00 \pm 1,24$ & $13,56 \pm 1,47 *$ \\
Eritrócitos / mm & $4577273 \pm 261737$ & $4668182 \pm 349675 *$ \\
\hline
\end{tabular}

* diferença com valor de $\mathrm{p}<0,05$ 
TABELA IV - Valores relativos à pressão arterial e potência aeróbica antes e após o programa $(\mathrm{n}=22)$.

\begin{tabular}{lcc}
\hline & $\begin{array}{c}\text { Valores iniciais } \\
(\text { média } \pm \mathrm{DP})\end{array}$ & $\begin{array}{c}\text { Valores finais } \\
(\text { média } \pm \mathrm{DP})\end{array}$ \\
\hline FC repouso $(\mathrm{bpm})$ & $85,29 \pm 12,30$ & $84,33 \pm 8,39$ \\
PAS repouso $(\mathrm{mmHg})$ & $131,68 \pm 17,39$ & $130,90 \pm 15,16$ \\
PAD repouso $(\mathrm{mmHg})$ & $92,27 \pm 15,11$ & $87,72 \pm 10,97$ \\
$\mathrm{VO}_{2}$ pico $\left(\mathrm{ml} . \mathrm{kg} \cdot \mathrm{min}^{-1}\right)$ & $19,88 \pm 3,47$ & $23,02 \pm 4,33 *$ \\
\hline
\end{tabular}

$* \mathrm{p}<0,05$; FC: freqüência cardíaca; PAS: pressão arterial sistólica; PAD: pressão arterial diastólica; $\mathrm{VO}_{2 \text { pico }}$ : consumo pico de oxigênio.

2000). Outro resultado que merece destaque é o aumento da hemoglobina e dos eritrócitos, cujo papel é importante no transporte de oxigênio dos pulmões para os tecidos (Lorenzi, Verrastro, 1999). Dentre várias respostas adaptativas ao treinamento físico, uma delas é o aumento da volemia e conseqüentemente da albumina e das hemácias. O treinamento também proporciona aumento da capilarização dos músculos. Desta forma, o aumento da capacidade de consumir oxigênio, paralelo ao aumento dos mecanismos de transporte e captação celular do oxigênio, resultam em maior eficiência para realização do exercício, tendo como resultado final a melhoria do condicionamento físico (Foss, Keteyian, 2000; Slentz et al., 2004; Ross et al., 2000).

O treinamento físico realizado no presente estudo combinou atividades aeróbias e de resistência muscular, devido às vantagens específicas de cada tipo de exercício, principalmente em se tratando de indivíduos obesos (ADES et al., 1996; ACSM, 2001; Braith, Stewart, 2006). Sarsan et al. (2006) submeteram 60 mulheres obesas a diferentes tipos de exercício: aeróbio e anaeróbio, comparativamente a um grupo controle. Os autores observaram melhoras em condições cardiovasculares e respiratórias em ambos os tipos de exercício, fato que comprova que a somatória dessas atividades pode trazer grandes benefícios aos praticantes.

Observando as variáveis antropométricas, pode-se inferir que o exercício, isoladamente, não promove perda de gordura subcutânea. Contudo, o resultado mais significativo nessa pesquisa refere-se à diminuição da obesidade central, considerada a mais relacionada ao desenvolvimento de doenças crônicas. Verificamos que houve redução significativa nas circunferências da cintura $(\mathrm{CC})$ e do quadril (CQ), e também na relação cintura/quadril (RCQ). Estes resultados estão de acordo com os de Lee et al. (2005), que ao final de três meses de exercício mistos, sem alteração drástica na ingestão energética, encontraram que todos os avaliados tiveram redução da gordura visceral, sem alteração do IMC.
A CC é considerada um bom preditor para mudanças na gordura intra-abdominal durante a perda de massa corporal, pois reflete a proporção de gordura localizada nessa região e correlaciona-se bem com medidas de adiposidade usando ressonância magnética ou tomografia computadorizada (Lean, Han, Morrison, 1995; Han et al., 1995). No presente estudo observou-se uma diminuição significativa da circunferência da cintura (CC). Por tratar-se de uma medida simples, a CC é amplamente utilizada na literatura para avaliar adiposidade visceral, havendo sugestões de pontos de corte associados ao risco de desenvolver doenças crônicas (Ribeiro Filho et al., 2006). O National Cholesterol Education Program considera valores de $\mathrm{CC}$ de $88 \mathrm{~cm}$ para mulheres associados ao risco elevado de doenças cardiovasculares (Executive Summary of the third report of the National Cholesterol Education Program - NCEP-ATP III, 2001). De acordo com esses valores préestipulados, as mulheres que participaram do nosso estudo permanecem sob risco, mas podemos considerar que em apenas 12 semanas de exercícios, o programa trouxe efeitos benéficos para a saúde das mulheres pela diminuição significativa dessa variável. Por sua vez, com respeito à relação cintura-quadril (RCQ), o ponto de corte é 0,88 (Lean, Han, Morrison, 1995), e portanto a RCQ não apontou risco para essas mulheres nem no início e nem no final do programa.

Um estudo epidemiológico realizado em São Paulo-SP com 1.402 indivíduos, procurou identificar variáveis associadas com as medidas de CC e RCQ, na perspectiva de eleger a melhor delas. Foi verificado que a RCQ guardou maior associação com eventos passados como desnutrição, ou ainda hábitos de vida como sedentarismo, fumo entre outros. Por outro lado, a circunferência da cintura se associou melhor com dados bioquímicos de dislipidemias (Martins, Marinho, 2003). Chan et al. (2003), ao estudar 59 indivíduos do sexo masculino, relacionaram as duas medidas com parâmetros de gordura visceral a partir de ressonância magnética nuclear. Os autores atribuíram à circunferência da cintura o melhor indicador de gordura visceral.

A lipólise em humanos tem sido descrita como uma forte resposta à ação das catecolaminas nas células adiposas do tecido adiposo visceral. De modo geral, o tecido adiposo visceral é o mais ativo, ou seja, mais sensível à lipolise (via catecolaminas e $\beta$-adrenorreceptores) do que o tecido adiposo subcutâneo e glúteo femural (Kelley et al., 2000). Desta forma, a diminuição da CC pode ser explicada possivelmente pelo efeito do exercício físico na liberação da adrenalina (Wajchenberg, 2000). Por sua vez, é importante relatar estudos que afirmam que a gordura presente na CQ (tecido adiposo subcutâneo e glúteo femural) expressa maior concentração de algumas citocinas em relação ao tecido adiposo visceral. Um exemplo dessas citocinas seria a ASP (proteína estimulante de 
acilação), que tem a capacidade de inibir a lipólise e estimular a lipogênese. Isto explicaria de alguma forma a relação dessa citocina com a síndrome metabólica, e também justificaria a medida da CQ na avaliação do risco de doenças crônicas (Rajala et al., 2004).

A CC se correlacionou inversamente com o consumo pico de oxigênio $(r=-0,52 ; p=0,013)$, demonstrando que o condicionamento cardiovascular proporcionado pelo treinamento físico tem influência na distribuição da gordura corporal. Resultados similares foram encontrados por Lee et al. (2005) que verificaram que um bom condicionamento cardiovascular é relacionado com menor risco metabólico. Essa relação ainda não é totalmente compreendida, mas os autores sugerem que o $\mathrm{VO}_{2}$ reflete modelos recentes de atividade física dos participantes e que menores níveis de pressão arterial e lipídeos plasmáticos são normalmente associados a uma rotina de atividades físicas, podendo explicar parcialmente tais resultados.

Outro resultado importante do presente estudo, também relacionado ao risco de desenvolver doenças crônicas, foi a fração lipoprotéica de alta densidade (HDL-colesterol) que teve um significativo aumento após o programa. Essa melhora no HDL-colesterol é atribuída também aos efeitos do exercício físico (Hardman, 1999). Couillard et al. (2001) verificaram que o exercício físico regular (particularmente aeróbico) ajuda a melhorar o perfil lipoprotéico de homens com baixo HDL-colesterol, obesidade abdominal e altos níveis de triglicerídeos. Zmuda et al. (1998) compararam os efeitos de um programa de exercícios físicos, com duração de 12 meses, nos níveis de HDL de homens com concentrações iniciais normais e baixas. Verificaram que os indivíduos com baixo HDL inicial, tem habilidade limitada para alterar o metabolismo dos triglicerídeos e aumentar os níveis de HDL quando comparados aos sujeitos com níveis normais de HDL no início do programa. Os índices I e II de Castelli apresentaram modesta redução, porém sem diferença estatisticamente significante. Tanto nos valores iniciais quanto após o programa, as mulheres estavam com risco aumentado para desenvolvimento de doenças cardiovasculares, de acordo com a classificação da Sociedade Brasileira de Angiologia e de Cirurgia Vascular (Duque, 1998). Provavelmente um tempo maior de treinamento físico poderia ter demonstrado significância nesses valores.

No presente estudo, a glicemia plasmática na situação de jejum também sofreu redução significativa, situando os valores médios das mulheres abaixo do ponto de corte estabelecido pela Sociedade Brasileira de Diabetes (SBD, 2005; $<100 \mathrm{mg} / \mathrm{dL}$ ). Resultados similares foram encontrados por Silva, Lima (2002) que estudaram 33 sujeitos diabéticos que foram submetidos a 10 semanas de exercícios aeróbicos e de resistência muscular localizada. Ao final do estudo, obser- vou-se que o exercício apresentou um efeito benéfico com relação à glicemia, de forma aguda e crônica. Ou seja, logo após uma única sessão de exercício, a glicemia capilar teve uma diminuição média de $31 \mathrm{mg} / \mathrm{dL}$ e após as 10 semanas, a glicemia de jejum diminuiu em média $8,4 \mathrm{mg} / \mathrm{dL}$. Ross et al. (2004) verificaram que o exercício físico sem restrição calórica foi associado a reduções significativas na gordura total, gordura abdominal e visceral e resistência a insulina em mulheres obesas. Apesar da nossa amostra não apresentar sujeitos diabéticos, tais estudos comprovam as alterações benéficas que o exercício proporciona ao metabolismo da glicose tanto em sujeitos diabéticos quanto obesos.

A partir dos resultados encontrados no presente estudo, pode-se concluir que o exercício físico demonstrou-se uma importante estratégia para reduzir os fatores de risco para desenvolvimento de doenças crônicas em mulheres obesas. Por outro lado, 12 semanas de treinamento possivelmente não foram suficientes para que fossem observadas outras alterações, especialmente relacionadas à composição corporal.

\section{ABSTRACT}

\section{Effect of physical exercise on risk factors for chronic diseases in obeses women}

This study aimed to evaluate the effects of physical exercise on body weight reduction. For 12 weeks, 22 obese women $\left(B M I>30 \mathrm{~kg} / \mathrm{m}^{2}\right)$ were submitted to a physical exercise program. At the beginning and at the final of the program there were evaluated: BMI, waist (WC) and hip circumferences (HC), and waist-hip ratio (WHR); body composition by DEXA; hemoglobin and erythrocyte, total cholesterol, HDL and LDL, triacylglycerol and blood glucose; aerobic power. At the final of the program, aerobic power, hemoglobin and erythrocyte values were significantly increased, confirming the physical training effects. Related to anthropometric values, only the visceral fat (WC, HC and WHR) were reduced. The exercise shows to be an important supporting in the body weight loss program, not exactly promoting body weight loss, but lowering risk factors to develop chronic diseases.

UNITERMS: Obesity. Physical exercise/effects. Chronic diseases.

\section{AGRADECIMENTOS}

Os autores expressam seus agradecimentos à FAPESP pelo financiamento do projeto de pesquisa (Processo 03/08986-0), ao CNPq e CAPES pelas bolsas de estudos e ao Laboratório Rhesus-Medicina Diagnóstica, por possibilitar as avaliações por DEXA. 


\section{REFERÊNCIAS BIBLIOGRÁFICAS}

ACSM - American College of Sports Medicine. ACSM Position stand on appropriate intervention strategies for weight loss and prevention of weight regain for adults. Med. Sci. Sports. Exerc., v.33, p.2145-2156, 2001.

ADA - American Dietetic Association. Position of the American Dietetic Association: Weight Management. $J$. Am. Diet. Assoc., v.102, p.1145-1155, 2002.

BARBATO, K.B.G.; MARTINS, R.C.V.; RODRIGUES, M.L.G.; BRAGA, J.U.; FRANCISCHETTI, E.A.; GENELHU, V.Efeito da redução de peso superior a 5\% nos perfis hemodinâmico, metabólico e neuroendócrino de obesos Grau I. Arq. Bras. Cardiol., v.87, p.12-21, 2006.

BORG, G.A.V.Physiological bases of perceived exertion. Med. Sci. Sports. Exerc., v.14, p.377-387, 1982.

BRAITH, R.W.; STEWART, K.J. Resistance exercise training: its role in the prevention of cardiovascular disease. Circulation, v.113, p.2642-2650, 2006.

CASTELLI, W.P.; GARRISON, R.J.; WILSON, P.W.F.; ABBOT, R.D.; KALOUSDIAN, S.; KANNEL, W.N. Incidence of coronary heart disease and lipoprotein cholesterol levels: the Framingham Heart Study. JAMA, v.256, p.2835-2838, 1986.

CHAN, D.C.; WATTS, G.F.; BARRETT, P.H.R.; BURKE, V.Waist circumference, waist-to-hip ratio and body mass index as predictor os adipose tissue compartments in men. Q. J. Med., v.96, p.441-447, 2003.

COUILLARD, C.; DESPRÉS, J.P.; LAMARCHE, B.; BERGERON, J.; GAGNON, J.; LEON,A.S.; RAO, D.C.; SKINNER, J.S.; WILMORE, J.H.; BOUCHARD, C. Effects of endurance exercise training on plasma HDL cholesterol levels depend on levels of triglycerides: evidence from men of the health, risk factors, exercise training and genetics (HERITAGE) family study. Arterioscler. Thromb. Vasc. Biol., v.21, p.1226-1232, 2001.

DUQUE, F.L.V.Aterosclerose: aterogênese e fatores de risco. Rev.Angiol. Cir. Vasc., v.7, p.100-116, 1998.

FOSS, M.L.; KETEYIAN, S.J. Bases fisiológicas do exercício e do esporte. 6.ed. Rio de Janeiro: Koogan, 2000. p.184-187.
HAN, T.S.; LEER, E.M.; SEIDELL, J.C.; LEAN, M.E.J. Waist cincunferemce action level in the identification for cardiovascuar risk factor: prevalence study in a randam sample. $B M J$, v.311, p.1401-1405, 1995.

HARDMAN, A.E. Physicalactivity, obesity and blood lipids. Int. J. Obes., v.23, suppl. 3, p.S64-S71, 1999.

HEDLEY, A.A.; OGDEN, C.L.; JOHNSON, C.L.; CARROLL, M.D.; CURTIN, L.R.; FLEGAL, K.M. Prevalence of overweight and obesity among US children, adolescents and adults, 1999-2002. JAMA, v.291, p.28472850, 2004

IBGE. Instituto Brasileiro de Geografia e Estatística. Diretoria de Pesquisas. Coordenação de Índices de Preços. Pesquisa de Orçamentos Familiares. Brasília, 2002-2003. 270p.

KELLEY, D.E.; THAETE, F.L.; TROOST, F.; HUWE, T.; GOODPASTER, B.H. Subdivisions of subcutaneous abdominal adipose tissue and insulin resistance. Am. J. Physiol. Endocrinol. Met., v.278, p.E941-E948, 2000.

KEMPEN, K.P.G.; SARIS, W.H.M.; WESTERTERP, K.R. Energy Balance During an 8-wk energy restricted diet with and without exercise in obese women. Am. J. Clin. Nutr., v.62, p.722-729, 1995.

LEAN, M.E.J.; HAN, T.S.; MORRISON, T.E. Waist Circumference as a measure for indicating need for weight management. BMJ, v.311, p.158-161, 1995.

LEE, S.J.; KUK, J.L.; DAVIDSON, L.E.; HUDSON, R.; KILPATRICK, K.; GRAHAM, T.E.; ROSS, R. Exercise without weight loss is an effective strategy for obesity reduction in obese individuals with and without Type 2 diabetes. J. Appl. Physiol., v.99, p.1220-1225, 2005.

LEE, S.J.; KUK, J.L.; KATZMARZYK, P.T.; BLAIR, S.N.; CHURCH, T.S.; ROSS, R. Cardiorespiratory fitness attenuates metabolic risk independent of abdominal subcutaneous and visceral fat in men. Diabetes Care, v.28, p.895-901, 2005.

LORENZI, T.F.; VERRASTRO, T. Eritrócitos. In: AIRES, M. M. Fisiologia. 2.ed. Rio de Janeiro: Koogan, 1999. p.107-117.

MARTINS, I.S.; MARINHO, S.P.O potencial diagnóstico dos indicadores da obesidade centralizada. Rev.Saúde Publica, v.37, p.760-767, 2003. 
MONTEIRO, R.C.A.; RIETHER, P.T.A.; BURINI, R.C. Efeitos de um programa misto de intervenção nutricional e exercício físico sobre a composição corporal e os hábitos alimentares de mulheres obesas em climatério. Rev. Nutr., v.17, p.479-489, 2004.

NCEP-ATPIII - Executive summary of the Third Report of the National Cholesterol Education Program (NCEP) Expert Panel on Detection, Evaluation and Treatment of High Blood Cholesterol in Adults (Adult Treatment Panel III). JAMA, v.285, p.2486-2497, 2001.

OKURA, T.; TANAKA, K.; NAKANISHI, T.; LEE, D.J.; NAKATA, Y.; WEE, S.W.; SHIMOKATA, H. Effects of obesity phenotype on coronary heart disease risk factor in response to weight loss. Obes. Res., v.10, p.757-766, 2002.

PAFFENBARGER, R.S.; BLAIR, S.N.; LEE, I.M.; HYDE, R.T. Measurement of physical activity to assess health effects in free-living populations. Med. Sci. Sports Exerc., v.25, p.60-70, 1993.

PIETROBELLI, A.; FORMICA, C.; WANG, Z.M.; HEYMSFIELD, S.B. Dual-energy X-ray absorptiometry body composition model: Review of physical concepts. Am. J. Physiol., v.271, p.E941-E951, 1996.

RAJALA, M.W.; QI, Y.; PATEL, H.R.; TAKAHASHI, N.; BANERJEE, R.; et al. Regulation of resistin expression and circulating levels in obesity, diabetes, and fasting. Diabetes, v.53, p.1671-1679, 2004.

RIBEIRO FILHO, F.F.; MARIOSA, L.S.; FERREIRA, S.R.G.; ZANELLA, M.T. Gordura Visceral e Síndrome Metabólica: mais do que uma simples associação. Arq. Bras. Endocrinol. Met., v.50, p.230-238, 2006.

RIPPE, J.M. The case for Medical Management of Obesity: a call for increased physician involvement. Obes. Res., v.6, suppl. 1, p.23S-33S, 1998.

ROSS, R.; DAGNONE, D.; JONES, P.J.H.; SMITH, H.; PADDAGS, A.; HUDSON, R.; JANSSEN, I. Reduction in obesity and related comorbid conditions after dietinduced weight loss ou exercise-induced weight loss in men. Ann. Intern. Med., v.133, p.92-103, 2000.
ROSS, R.; JANSSEN, I.; DAWSON, J.; KUNGL, A.M.; KUK, J.L.; WONG, S.L.; NGUYEN-DUY, T.B.; LEE, S.J.; KILPATRICK, K.;HUDSON, R. Exercise-induced reduction in obesity and insulin resistance in women: a randomized controlled trial. Obes. Res., v.12, p.789-798, 2004.

SARSAN, A.; ARDIC, F; OZGEN, M.; TOPY, O.; SERMEZ, W. The effects of aerobic and resistance exercise in obese women. Clin. Rehabil., v.20, p.773-782, 2006.

SBD. Sociedade Brasileira de Diabetes: Atualização brasileira sobre diabetes. Rio de Janeiro: Diagraphic, 2005. 140p.

SILVA, C.A.; LIMA, W.C. Efeito benéfico do exercício no controle metabólico do diabetes mellitus tipo 2 à curto prazo. Arq. Bras. Endocrinol. Met., v.46, p.550-556, 2002.

SLENTZ, C.A.; DUSCHA, B.D.; JOHNSON, J.L.; KETCHUM, K.; AIKEN, L.B.; SAMSA, G.P.; HOUMARD, J.A.; BALES, C.W.; KRAUS, W.E. Effects of the amount of exercise on body weight, body composition, and measures of central obesity. Arch. Intern. Med., v.164, p.31-40, 2004.

UAUY, R.; ALBALA, C.; KAIN, J. Obesity Trends in Latin America: Transition from Under - to Overweight. J. Nutr., v.131, p.893S-899S, 2001.

WAJCHENBERG, B.E. Subcutaneous and visceral adipose tissue: their relation to the metabolic syndrome. Endocrine rev., v.21, p.697-738, 2000.

WHO. World Health Organization. Obesity: preventing and managing the global epidemic. Report of a WHO consultation on obesity. Geneva, 1997.251p.

ZMUDA, J.M.; YURGALEVITCH, S.M.; FLYNN, M.M.; BAUSSERMAN, L.L.; SARATELLI, A.; SPANNAUSMARTIN, D.J.; HERBERT, P.N.; THOMPSON, P.D. Exercise training has little effect on HDL levels and metabolism in men with initially low HDL cholesterol. Atherosclerosis, v.137, p.215-221, 1998.

Recebido para publicação em 22 de agosto de 2007 Aceito para publicação em 12 de dezembro de 2007 